\title{
Transient Diabetes Insipidus during Pregnancy
}

Key words: vasopressin, MRI

The metabolism of water during pregnancy is remarkably changed by hormonal and hemodynamic mechanisms. In pregnant women, total body water usually increases nearly 7 liters and plasma volume expands more than 1.4 times that of non-pregnant women. The secretion of the antidiuretic hormone, vasopressin, which is the most important hormone in the metabolism of water, is also affected by pregnancy. First, plasma osmolality physiologically decreases $8-10 \mathrm{mOsm} / \mathrm{kg}$ below the level of women who are not pregnant, suggesting that plasma vasopressin might be suppressed because of hypo-osmolality. However, the osmolar threshold of vasopressin secretion shifts to a level lower than that of nonpregnant subjects, this change forms a new steady state in the regulation of vasopressin release (1). The mechanism of this adaptation is likely to be based on hormonal change, especially chorionic gonadotropin produced from placenta, but the details of this are not completely known at the time of this writing. Second, the metabolic clearance rate of vasopressin is accelerated approximately four-fold by several mechanisms. There is no specific enzyme to degradate vasopressin in non-pregnant subjects, however, oxytocinase (aminopeptidase) is significantly produced from the placenta during pregnancy. Oxytocinase inactivates not only oxytocin but also vasopressin, based upon the similar amino acid structure of both hormones. Moreover, the increase in blood circulation in the liver and kidneys also increases vasopressin clearance.

These physiological changes sometimes induce specific and transient disorders in water metabolism, such as diabetes insipidus during pregnancy (2). The increases in oxytocinase activity might modify vasopressin metabolism in even healthy pregnant women, as mentioned below. Therefore, it is possible that the patients with partial central diabetes insipidus, who do not show typical symptoms of severe polyuria or polydipsia because of incomplete but minimal secretion of vasopressin in the non-pregnant state, plasma vasopressin is readily degradated by oxytocinase to induce overt polyuria and polydipsia during pregnancy. To correctly evaluate plasma vasopressin during pregnancy, the procedure to inactivate oxytocinase using phenanthroline in collected blood samples is necessary.

In rare circumstances, extremely elevated levels of oxytocinase degradate plasma vasopressin and even normal pregnant women might manifest central diabetes insipidus.
Although reported cases of this disorder have gradually increased since the detailed report by Durr et al (3), to measure oxytocinase activity correctly as same as the evaluation of vasopressin release ability is the utmost importance in reaching the final diagnosis of this extraordinary state.

Recently, patients with autoimmune hypophysitis have been reported from many countries since the entity of infundibuloneurohypophysitis was proposed in 1993 (4). Autoimmune hypophysitis is generally divided into two forms, one is anterior hypophysitis and the other is infundibuloneurohypophysitis. The former is closely correlated with gestation and the latter shows no such apparent relationship with pregnancy. However, some cases were reported in which central diabetes insipidus was due to infundibuloneurohypophysitis in pregnancy. Therefore this possibility should not be excluded when transient diabetes insipidus is seen.

Generally, transient diabetes insipidus in pregnancy is initially found by clinical symptoms as polyuria and polydipsia. Diagnostic process is not easy since water metabolism changes so much, as mentioned above. The first diagnostic step is to confirm free water diuresis in order to exclude osmotic diuresis in the case of diabetes mellitus. Next, the hypothalamic area and pituitary are observed by magnetic resonance images (MRI) to rule out some organic lesions. Central diabetes insipidus is generally caused by some brain tumor, trauma, post-operation, granuloma and inflammation. Although these diseases and changes rarely occur during pregnancy, it is always fundamental to exclude organic diseases to diagnose central diabetes insipidus (5). Characteristic change in the patients with central diabetes insipidus is usually seen as the absence of hyperintensity in the posterior pituitary by T1-weighted MRI, this has been found in both pregnant and non-pregnant patients. In this volume, Yamamoto et al (6) reported a patient with transient diabetes insipidus during pregnancy.

See also p 513.

The hyperintensity of the posterior pituitary in MRI was absent in gestation but it recovered to normal level after delivery, suggesting some etiological possibility for the clue of the precise mechanism in transient diabetes insipidus during pregnancy. Laboratory tests are also performed to measure basal plasma vasopressin, plasma osmolality and serum sodium for the functional diagnosis. Finally, DDAVP, a synthesized analogue of vasopressin, is administered for the purpose of therapeutic diagnosis. Fortunately, DDAVP is 
effective for the treatment in central diabetes insipidus during gestation because oxytocinase does not breakdown DDAVP.

Moreover, transient nephrogenic diabetes insipidus also manifests itself during pregnancy. The concentration of prostaglandin E2 in the kidney physiologically increases during gestation and this increase lowers vasopressin sensitivity in the collecting duct of the kidney.

Polyuria during pregnancy arises under such a complicated and varied pathological basis, therefore we should diagnose and treat correctly to maintain normal conditions during pregnancy and for the healthy development of fetus.

Yutaka OISO, MD, PhD

Division of Metabolic Diseases, Department of Internal Medicine, Nagoya University Graduate School of Medicine, 65 Tsurumaicho, Showa-ku, Nagoya 466-8550

\section{References}

1) Lindheimer MD, Davison JM. Osmoregulation, the secretion of arginine vasopressin and its metabolism during pregnancy. Eur $\mathrm{J}$ End 132: 133-143, 1995.

2) Iwasaki Y, Oiso Y, Kondo K, et al. Aggravation of subclinical diabetes insipidus during pregnancy. New Engl J Med 324: 522-526, 1991.

3) Durr JA, Hoggard JG, Hunt JM, Schrier RW. Diabetes insipidus in pregnancy associated with abnormally high circulating vasopressinase activity. New Engl J Med 316: 1070-1074, 1987.

4) Imura $H$, Nakao $K$, Shimatsu $A$, et al. Lymphocytic infundibuloneurohypophysitis as a cause of central diabetes insipidus. New Engl $\mathbf{J}$ Med 329: 683-689, 1993.

5) Hashimoto M, Ogura T, Otsuka F, et al. Manifestation of subclinical diabetes insipidus due to pituitary tumor during pregnancy. Endocr $\mathbf{J}$ 43: 577-583, 1996.

6) Yamamoto $T$, Ishii $T$, Yoshioka $K$, et al. Transient central diabetes insipidus in pregnancy with a peculiar change in signal intensity on T1weighted magnetic resonance images. Intern Med 42: 513-516, 2003. 